This is a self-archived - parallel published version of this article in the publication archive of the University of Vaasa. It might differ from the original.

\title{
Flexible co-operation of TCSC and corrective topology control under wind uncertainty : an interval-based robust approach
}

Author(s): Nikoobakht, Ahmad ; Aghaei, Jamshid ; Lotfi, Mohamed ; Catalão, João P.S. ; Osório, Gerardo J. ; Shafie-khah, Miadreza

Title: $\quad$ Flexible co-operation of TCSC and corrective topology control under wind uncertainty : an interval-based robust approach

Year: $\quad 2019$

Version: Accepted manuscript

Copyright (C)2019 IEEE. Personal use of this material is permitted. Permission from IEEE must be obtained for all other uses, in any current or future media, including reprinting/republishing this material for advertising or promotional purposes, creating new collective works, for resale or redistribution to servers or lists, or reuse of any copyrighted component of this work in other works.

\section{Please cite the original version:}

Nikoobakht, A., Aghaei, J., Lotfi, M., Catalão, J.P.S., Osório, G.J., Shafie-khah, M., (2019). Flexible co-operation of TCSC and corrective topology control under wind uncertainty : an intervalbased robust approach. In: IEEE Milan PowerTech, Milan, Italy. https://doi.org/10.1109/PTC.2019.8810889 


\section{Flexible Co-Operation of TCSC and Corrective Topology Control under Wind Uncertainty: An Interval-based Robust Approach}

\author{
Ahmad Nikoobakht \\ Higher Education \\ Center of Eghlid, \\ Eghlid, Iran

\begin{abstract}
Jamshid Aghaei
Shiraz University

of Technology,
\end{abstract} \\ Shiraz, Iran \\ a.nikoobakht@eghlid.ac.ir aghaei@sutech.ac.ir
}

\author{
Mohamed Lotfi, João P. S. Catalão \\ FEUP and INESC TEC, \\ Porto 4200-465, Portugal \\ mohd.f.lotfi@gmail.com; \\ catalao@fe.up.pt
}

\author{
Gerardo J. Osório Miadreza Shafie-khah \\ C-MAST/UBI, \\ Covilha, Portugal \\ gjosilva@gmail.com
}

\begin{abstract}
This paper presents an AC optimal power flow (ACOPF) model including flexible resources (FRs) to handle uncertain wind power generation (WPG). The FRs considered are thermal units with up/down re-dispatching capability, corrective topology control (CTC), and thyristor-controlled series capacitors (TCSC). WPG uncertainty has been modeled through a proposed interval-based robust approach, the goal of which is to maximize the variation range of WPG uncertainty in power systems while maintaining an adequate reliability level at a reasonable cost with the aid of FRs. However, utilization of FRs (especially CTC and TCSC devices) is limited due to the difficulty of their incorporation in the $\mathrm{AC}-\mathrm{OPF}$. The optimization framework of the full FR-augmented AC-OPF problem is a mixed-integer nonlinear programming (MINLP) in which the solution for large-scale systems is very hard to obtain. To solve this issue, this paper uses a two-stage decomposition algorithm to decompose the MINLP representation into a mixed-integer linear program (MILP) and a nonlinear program (NLP). Finally, the robust AC-OPF model with FRs is implemented and tested on a 6-bus and the IEEE 118-bus test systems to evaluate its efficiency and performance.
\end{abstract}

Index Terms-AC Optimal Power Flow, Thyristor controlled series capacitor, corrective topology control.

\begin{tabular}{|c|c|}
\hline \multirow{2}{*}{\multicolumn{2}{|c|}{ NOMENCLATURE }} \\
\hline & \\
\hline$w, g$ & Wind farm and Generation units. \\
\hline$n / m$ & System buses. \\
\hline$k$ & Transmission line. \\
\hline$(\cdot)^{ \pm}$ & $\begin{array}{l}\text { Wind uncertainty: "+" is upper and "-" is lower boundary } \\
\text { of wind uncertainty, }(\cdot)^{0} \text { relate first stage and }(\cdot)^{ \pm} \text {relate } \\
\text { to second stage variable (uncertainty condition). }\end{array}$ \\
\hline \multicolumn{2}{|l|}{ Sets } \\
\hline$\chi_{n}$ & Set of generation units connected to bus $n$. \\
\hline$\Omega_{n}$ & Set of transmission lines connected to bus $n$. \\
\hline \multicolumn{2}{|c|}{ Continuous Variables } \\
\hline$\varphi_{n m}^{(\cdot)}$ & Difference of voltage angles across line $(n, m)$. \\
\hline$P_{g}^{(\cdot)} / Q_{g}^{(\cdot}$ & Real/ reactive generation of unit $g$. \\
\hline$V_{n}^{(\cdot)}$ & Voltage magnitude at bus $n$. \\
\hline
\end{tabular}

$f_{n m}^{(\cdot)} / \tilde{f}_{n m}^{(\cdot)}$
$F_{n m}^{(\cdot)} / \tilde{F}_{n m}^{(\cdot)}$
$f_{V R D, n m}^{(\cdot)} / \tilde{f}_{V R D, n m}^{(\cdot)}$
$\bar{\Delta}_{g}^{(\cdot)} / \Delta r_{g}^{(\cdot)}$
$x_{k}^{V R D}$
$\bar{g}_{k} / \bar{b}_{k}$
$T C_{b} / T C$
$\lambda$
$\kappa_{(\bullet)}^{(\cdot)}$
$\Phi$
Binary Variables
$v_{k}$
$\vartheta_{k}^{(}$

\section{Constants}

$\bar{\varphi}_{n m} / \varphi_{n m}$

$\bar{x}_{k} / \underline{x}_{k}$

$c_{g}^{ \pm} / c_{g}$

$S_{k}^{\max }$

$\psi_{D_{n}}$

$P_{f, w}$

$\Delta \Phi_{g}^{ \pm}$

$q_{g}^{\max } / q_{g}^{\min }$

$d_{n}$

M

$g_{k} / b_{k}$

$b_{k_{0}}$

$\xi$
Real/reactive power flow from bus $n$ to bus $m$.

Real/reactive power flow from bus $n$ to bus $m$ of line $(n, m)$ equipped with VRD.

Real power increase/decrease in unit $g$ for security purposes.

Reactance of the VRD installed on line $k$.

Conductance/ series admittance of line $k$.

Total cost for base case/uncertainty case.

Variation of wind uncertainty.

Dual variable.

Active power mismatch.

Switching state of line $k$.

Binary variable implying whether TCSC is placed on line $k$ or not.

Max/min difference of voltage angle across line $(n, m)$.

Max/min reactance change of TCSC for line $k$

Cost of wind uncertainty / normal conditions of generation unit $g$.

Maximum amount of MVA flow through line $k$

Power factor of demand $n$.

Forecasted power production of wind farm $w$.

Ramp up (down) limit of generation unit $g$ at wind uncertainty condition.

$\mathrm{Max} / \mathrm{min}$ reactive power generation of generation unit $g$.

Real power consumption via demand $n$.

A large number (positive).

Conductance/series admittance of line $k$.

Shunt admittance of transmission line $k$.

Critical tolerance level of cost threshold. 


\section{INTRODUCTION}

Nowadays, the contribution of wind power generators (WPGs) in power systems has progressively increased. However, the intrinsic uncertainty of WPGs as well as possible transmission congestion bottlenecks may hinder the integration of WPGs into power systems. Likewise, higher uncertainty in operation of power system, especially arisen by integrating WPGs, necessitates more flexibility to satisfy the load-generation balance. By increased uncertainty of WPG in power system operations, the balance of supply and demand can be potentially maintained by supply side (SS) flexibilities. However, the main obstacle in SS flexibility (or thermal unit redispatch) is transmission lines congestion.

Therefore, flexible AC transmission systems (FACTS) devices, like thyristor-controlled series capacitor (TCSC) and CTC, being the network side (NS) flexibility resources, can effectively enhance transfer capability and improve integration of REGs with enhanced utilization of SS flexibility [1]. In several OPF problems, the TCSC devices have been utilized to enhance transmission capacity and reduce transmission congestion [2], [3]. An optimal tuning methodology of TCSC settings has been proposed in [4] to have a better usage of the existing transmission system in the presence of wind uncertainty. This is the focus of this study, while corrective topology control (CTC) action has not been modelled in the current work [1], [5] and [6].

The aim of this paper is to maximize the probable variation range of WPG uncertainty that a power system can accommodate for a given system congestion condition, while maintaining power system security.

Also, uncertainty modeling is an important component in modern power systems and plays a key role in power system operation [7] and [6]. Accordingly, researchers have recently shown an increased interest in the use of stochastic programming (SP) to solve the OPF problem while considering different uncertainty sources [8]. However, the $\mathrm{SP}$ is based on the probability density functions (PDFs) of the uncertainty parameters (UPs) to create probable scenarios. This is challenging since generally it is difficult to extract PDFs for UPs in real-world power systems.

In this study an interval-based robust approach is utilized to model the WPG uncertainty. It does not require the PDF of UPs since it models the UPs considering the variation range of UPs. The proposed interval-based robust approach finds the largest variation range of uncertainty in a way the solution of the proposed optimization problem is immunized against the possible uncertainties.

Such an optimization problem with flexible resources (i.e., the TCSC device and CTC) is called generalized accurate OPF model. Hence, the proper modeling of the uncertain WPG in full AC-OPF formulation is crucial.

In most papers, the direct current OPF (DC-OPF) model, which does not consider neither voltage magnitude, reactive power, nor network losses, is implemented in the power system to model TCSC and CTC, which is only because of its speed and simplicity [9]. Nonetheless, modeling the CTC and TCSC device with DC-OPF equations is unacceptable for the following reasons: (i) The DC-OPF neglects transmission losses and reactive powers and may lead to an infeasible ACOPF solution, in this condition the security of power system may be endangered, especially, once implementing the CTC decisions [10], [11]. (ii) By modeling the CTC and TCSC device in DC-OPF cannot facilitate all benefits of the CTC and TCSC device, such as decreasing power grid losses or modifying voltage violations [10].

On the other hand, incorporating the CTC into the full AC-OPF involves the modeling of binary variables, which creates a mixed integer non-linear programming (MINLP) problem - a very complex problem to solve in reasonable time[12], [13]. Accordingly, to solve this challenge a Benders decomposition algorithm (BDA) is implemented to split the proposed problem into a master problem of the networkconstrained DC-OPF, which is mixed-integer linear program (MILP) problem, and several network-constrained AC-OPF sub-problems, which are MINLP problem.

Accordingly, the main contributions of this paper are summarized as follows:

- This paper develops an AC-OPF model incorporating TCSC and CTC to enhance utilization of WPG.

- A new interval-based robust method is proposed to identify worst-case uncertainty by means of bounded variation intervals instead of PDFs. Under the proposed robust framework, maximum possible uncertainty variation range that the system can accommodate is evaluated.

\section{MATHEMATICAL AC-OPF FORMULATION WITH FLEXIBLE RESOURCES}

In this section, the constraints of the original AC-OPF problem, along with the nonlinear TCSC device model and CTC, are described in detail. Accordingly, the AC-OPF problem with FRs is formulated as follows:

$\min T C=\sum_{g}\left(c_{g} P_{g}+c_{g}^{ \pm}\left(\left(\underline{\Delta} r_{g}^{-}+\bar{\Delta} r_{g}^{-}\right)+\left(\underline{\Delta} r_{g}^{+}+\bar{\Delta} r_{g}^{+}\right)\right)\right)$

Equation (1) is the objective function or total cost (TC) of the proposed problem, which subjects to the first stage constraints, i.e., (2)-(19), and second stage constraints, i.e., (20)-(38), respectively. The objective function (1) include two terms: The first term $\left(c_{g} P_{g}\right)$ related to cost of generation units at the normal condition, (or base case), and the second term $\left(c_{g}^{ \pm}\left(\left(\underline{\underline{\Delta}} r_{g}^{-}+\bar{\Delta} r_{g}^{-}\right)+\left(\underline{\underline{\Delta}} r_{g}^{+}+\bar{\Delta} r_{g}^{+}\right)\right)\right)$is related to the cost of corrective action to cover the lower and upper boundaries of largest variation range of wind uncertainty.

\section{First stage constraints}

$$
\begin{aligned}
& P_{g}^{\min } \leq P_{g} \leq P_{g}^{\max } \\
& q_{g}^{\text {min }} \leq q_{g} \leq q_{g}^{\max } \\
& \sum_{g \in \chi_{n}} P_{g}+\sum_{m \in \Omega_{n}}\left(\left(1-\vartheta_{k}\right) f_{n m}\right)+\sum_{m \in \Omega_{n}}\left(\vartheta_{k} f_{T C S C, n m}\right)+P_{f, w}=d_{n} \\
& \sum_{g \in \chi_{n}} q_{g}+\sum_{m \in \Omega_{n}}\left(\left(1-\vartheta_{k}\right) \tilde{f}_{n m}\right)+\sum_{m \in \Omega_{n}}\left(\vartheta_{k} \tilde{f}_{T C S C, n m}\right)=d_{n} \cdot \tan \left(\psi_{D_{n}}\right) \\
& F_{n m}=g_{k} V_{n}^{2}-V_{n} V_{m}\left(g_{k} \cos \varphi_{n m}+b_{k} \sin \varphi_{n m}\right)
\end{aligned}
$$




$$
\begin{aligned}
& \tilde{F}_{n m}=-\left(b_{k}+b_{k 0}\right) V_{n}^{2}+V_{n} V_{m}\left(b_{k} \cos \varphi_{n m}-g_{k} \sin \varphi_{n m}\right) \\
& F_{n m}-M\left(1-v_{k}\right) \leq f_{n m} \leq F_{n m}+M\left(1-v_{k}\right) \\
& \tilde{F}_{n m}-M\left(1-v_{k}\right) \leq \tilde{f}_{n m} \leq \tilde{F}_{n m}+M\left(1-v_{k}\right) \\
& -v_{k} f_{k}^{\max } \leq f_{n m} \leq v_{k} f_{k}^{\max } \\
& -v_{k} S_{k}^{\max } \leq \tilde{f}_{n m} \leq v_{k} S_{k}^{\max } \\
& \left(f_{n m}\right)^{2}+\left(\tilde{f}_{n m}\right)^{2} \leq v_{k}\left(S_{k}^{\max }\right)^{2} \\
& f_{T C S C, n m}=\left(-\left(\bar{b}_{k}+b_{k 0}\right) V_{n}^{2}+V_{n} V_{m}\left(\bar{b}_{k} \cos \varphi_{n m}-\bar{g}_{k} \sin \varphi_{n m}\right)\right) \\
& \tilde{f}_{T C S C, n m}=\left(-\left(\bar{b}_{k}+b_{k 0}\right) V_{n}^{2}+V_{n} V_{m}\left(\bar{b}_{k} \cos \varphi_{n m}-\bar{g}_{k} \sin \varphi_{n m}\right)\right) \\
& -f_{k}^{\max } \leq f_{T C S C, n m} \leq f_{k}^{\max } \\
& -S_{k}^{\max } \leq \tilde{f}_{T C S C, n m} \leq S_{k}^{\max } \\
& \left(f_{T C S C, n m}\right)^{2}+\left(\tilde{f}_{T C S C, n m}\right)^{2} \leq\left(S_{k}^{\max }\right)^{2} \\
& V_{n}^{\min } \leq V_{n} \leq V_{n}^{\max } \\
& \varphi_{k}^{\min } \leq \varphi_{n m} \leq \varphi_{k}^{\max }
\end{aligned}
$$

\section{Second-stage constraints}

$$
\begin{aligned}
& P_{g}^{ \pm}=\left(P_{g}^{0}+\left(\bar{\Delta} r_{g}^{+}-\underline{\Delta} r_{g}^{+}\right)+\left(\bar{\Delta} r_{g}^{-}-\underline{\Delta} r_{g}^{-}\right)\right) \\
& q_{g}^{\min } \leq q_{g}^{ \pm} \leq q_{g}^{\max } \\
& \sum_{g \in \chi_{n}} P_{g}^{ \pm}+\sum_{m \in \Omega_{n}}\left(\left(1-\vartheta_{k}\right) f_{n m}^{ \pm}\right)+\sum_{m \in \Omega_{n}}\left(\vartheta_{k} f_{T C S C, n m}^{ \pm}\right)+P_{w}^{ \pm}=d_{n} \\
& \sum_{g \in \chi_{n}} q_{g}^{ \pm}+\sum_{m \in \Omega_{n}}\left(\left(1-\vartheta_{k}\right) \tilde{f}_{n m}^{ \pm}\right)+\sum_{m \in \Omega_{n}}\left(\vartheta_{k} f_{T C S C, n m}^{ \pm}\right)=d_{n} \cdot \tan \left(\psi_{D_{n}}\right) \\
& F_{n m}^{ \pm}=g_{k} V_{n}^{ \pm}-V_{n}^{ \pm} V_{m}^{ \pm}\left(g_{k} \cos \varphi_{n m}^{ \pm}+b_{k} \sin \varphi_{n m}^{ \pm}\right) \\
& \tilde{F}_{n m}=-\left(b_{k}+b_{k 0}\right) V_{n}^{ \pm^{2}}+V_{n}^{ \pm} V_{m}^{ \pm}\left(b_{k} \cos \varphi_{n m}^{ \pm}-g_{k} \sin \varphi_{n m}^{ \pm}\right) \\
& F_{n m}^{ \pm}-M\left(1-v_{k}\right) \leq f_{n m}^{ \pm} \leq F_{n m}^{ \pm}+M\left(1-v_{k}\right) \\
& \tilde{F}_{n m}^{ \pm}-M\left(1-v_{k}\right) \leq \tilde{f}_{n m}^{ \pm} \leq \tilde{F}_{n m}^{ \pm}+M\left(1-v_{k}\right) \\
& -v_{k} f_{k}^{\max } \leq f_{n m}^{ \pm} \leq v_{k} f_{k}^{\max } \\
& -v_{k} S_{k}^{\max } \leq \tilde{f}_{n m}^{ \pm} \leq v_{k} S_{k}^{\max } \\
& \left(f_{n m}^{ \pm}\right)^{2}+\left(\tilde{f}_{n m}^{ \pm}\right)^{2} \leq v_{k}\left(S_{k}^{\max }\right)^{2} \\
& f_{V R D, n m}^{ \pm}=\bar{g}_{k}^{ \pm} V_{n}^{ \pm}-V_{n}^{ \pm} V_{m}^{ \pm}\left(\bar{g}_{k}^{ \pm} \cos \varphi_{n m}^{ \pm}+\bar{b}_{k}^{ \pm} \sin \varphi_{n m}^{ \pm}\right) \\
& \tilde{f}_{V R D, n m}^{ \pm}=-\left(\bar{b}_{k}^{ \pm}+b_{k 0}\right) V_{n}^{ \pm^{2}}+V_{n}^{ \pm} V_{m}^{ \pm}\left(\bar{b}_{k}^{ \pm} \cos \varphi_{n m}^{ \pm}-\bar{g}_{k}^{ \pm} \sin \varphi_{n m}^{ \pm}\right) \\
& -f_{k}^{\max } \leq f_{T C S C, n m}^{ \pm} \leq f_{k}^{\max } \\
& -S_{k}^{\max } \leq \tilde{f}_{T C S C, n m}^{ \pm} \leq S_{k}^{\max } \\
& \left(f_{V R D, n m}^{ \pm}\right)^{2}+\left(\tilde{f}_{T C S C, n m}^{ \pm}\right)^{2} \leq\left(S_{k}^{\max }\right)^{2} \\
& V_{n}^{\min } \leq V_{n}^{ \pm} \leq V_{n}^{\max } \\
& \varphi_{k}^{\min } \leq \varphi_{n m}^{ \pm} \leq \varphi_{k}^{\max } \\
& \left|P_{g}^{0}-P_{g}^{ \pm}\right| \leq \Delta \Phi_{g}^{ \pm} \\
&
\end{aligned}
$$

The limits on power active and reactive output of generation units is enforced by constraints (2) and (3), respectively. Constraints (4) and (5) represent the active and reactive power balance at each bus. Noted that, $\vartheta_{k}$ in (4) and (5) identify whether lines equipped with TCSC device or not. Therefore, $\vartheta_{k}$ is not a variable and it is an input parameter. Noted that $\vartheta_{k}=1$ implies a TCSC has been placed in line $k$, and otherwise $\vartheta_{k}=0$.

Constraints (6) and (7) correspond to active and reactive power flow equations without TCSC device, respectively. Constraint (8) is the CTC action formulation for active and reactive power flow [14]. The $M$ is necessarily large number.

The maximum active and reactive line flow rating for a closed line $k$ is defined by (10) and (11). Constraint (12) the MVA limit for line $k$. In constraints (8) - (12), once $v_{k}=1$, the line $k$ is closed or not switched on and once $v_{k}=0$ line $k$ is opened or switched off.

Constraints (13) and (14) correspond to active and reactive power flow equations of transmission lines equipped with TCSC devices, respectively. So, the $\bar{b}_{k}$ and $\bar{g}_{k}$ in (13) and (14) are variable and not parameter.

The constraints $(15)-(17)$ have the same expressions as (10) - (12), respectively, but these constraints correspond to lines that equipped with TCSC devices. Constraints (18) and (19) impose the lower and upper limits of voltage magnitudes and angles of buses.

Constraints (20) - (38) are related to second stage constraints. Where, in (20) - (38), "+"/ "-" relate to the minimum/maximum limits of the wind uncertainty intervals, respectively.

Equation (20) bundles between the base-case and secondstage (wind uncertainty) settings of a unit to apply corrective up/down re-scheduling actions, i.e., $\bar{\Delta} r_{g}^{ \pm} / \underline{\Delta} r_{g}^{ \pm}$.

Constraints $(21)-(37)$ are similar to (3) - (19) but related to wind uncertainty conditions, where the variables $P_{g}, q_{g}$, $F_{n m}, \tilde{F}_{n m}, f_{n m}, \tilde{f}_{n m}, \varphi_{n m}$ and $V_{n}$ are replaced by $P_{g}^{ \pm}, q_{g}^{ \pm}$, $F_{n m}^{ \pm}, \tilde{F}_{n m}^{ \pm}, f_{n m}^{ \pm}, \tilde{f}_{n m}^{ \pm}, \varphi_{n m}^{ \pm}, \bar{g}_{k}^{ \pm}, \bar{b}_{k}^{ \pm}$and $V_{n}^{ \pm}$, respectively. The changes in the power output of generation units for wind uncertainty condition is limited by ramp constraint (38). Where parameter $\Delta \Phi_{g}^{ \pm}$indicates the ramp rate of generation units in ten minutes to assure the desired security margin.

\section{INTERVAL-BASED ROBUST APPROACH}

The largest variation range of wind uncertainty can be is formulated as follows: $\max \lambda$

$P_{w}^{ \pm}=(1 \pm \lambda) P_{f, w}$

$T C \leq(1+\xi) T C_{b}$

The first stage constraints are:

(2) - (19)

The second stage constraints are :

(20) - (38)

The objective function (39) maximizes the span of the possible wind variation interval to find the worst-case uncertainty. 
The wind power generation (WPG) uncertainty condition has been shown by (39). In (40), the $\lambda$ value has a range between 0 to 1 that the minimum/maximum limits of possible WPG uncertainty correspond to the $(1+\lambda) P_{f, w}$ and $(1-\lambda) P_{f, w}$, respectively. The cost threshold, i.e., $(1+\xi) T C_{b}$, in equation (41) must not be surpassed for any realization of wind power uncertainty and it depends on the decision makers' conservatism level. The parameter, in constraint (41), is a positive value that set by the decision maker. Also, $T C_{b}$ is the total cost for the base cost threshold To specify this value the optimization problem (1) to (19) has been solved while $\lambda=0$, and the PFCTs are not considered, i.e., the value of $v_{k}$, (for TS action), and $x_{k}^{V R D}$ are fixed to zero. Noted that, here, it is supposed the AC-OPF problem, i.e., (2) - (19), without PFCTs, is certainly feasible for $\xi=0$.

\section{SOLUTION STRATEGY}

The original AC-OPF problem with the nonlinear TCSC device model and CTC which was modeled in section III is an MINLP problem. The main disadvantage of MINLP problem is that available solvers, e.g., DICOPT and BARON, for these problems cannot obtain global optimal solution with reasonable computational burden. To deal with this challenge, a Benders decomposition approach (BDA) [10] is implemented here to solve the proposed MINLP problem. As shown in Fig.1, the BDA include two parts: the first-part is a MILP problem indicated as master problem and the secondpart is a nonlinear sub-problem.

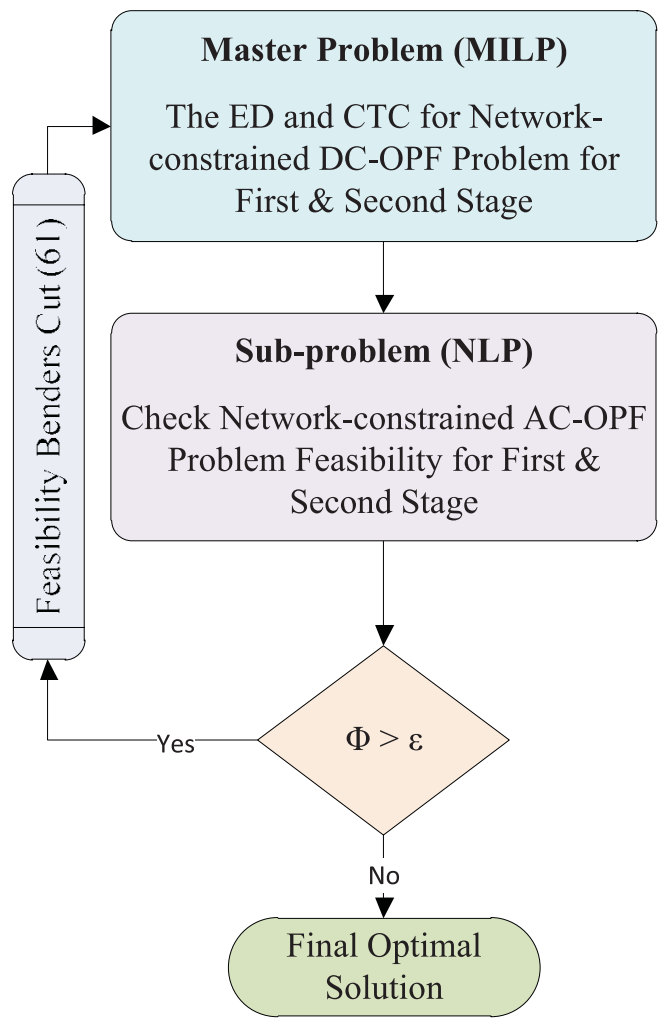

Fig. 1. The solution strategy based on BDA.
The master problem corresponds to DC-OPF problem including active power flow of the network while generally the reactive power and bus voltage limits are ignored in DC representation of the network. Then, the sub-problem checks the feasibility of AC-OPF constraints for the master problem solution. Then, the violations will be avoided with adjusting the set of lines to be switched or re-dispatching the power generation of the existing units as previously specified in the master problem. The detail formulations of the master problem and sub-problem are described as follows:

\section{A. Master problem}

The master problem is formulated as a networkconstrained DC-OPF problem that it is MILP problem. Here, the objective function (44) is optimized subject to the constraints (45)-(49).

$\max \lambda$

(40)-(41)

\section{The first stage constraints are:}

$b_{k} \varphi_{n m}-M\left(1-v_{k}\right) \leq f_{n m} \leq b_{k} \varphi_{n m}+M\left(1-v_{k}\right)$

(2), (4) (10), (15) and (19)

The second stage constraints are :

$b_{k} \varphi_{n m}^{ \pm}-M\left(1-v_{k}\right) \leq f_{n m}^{ \pm} \leq b_{k} \varphi_{n m}^{ \pm}+M\left(1-v_{k}\right)$

(20), (22), (26), (28), (33), (37) and (38)

Constraints (45), (47) and (49) have been mentioned in sections III and IV. Constraints (46) and (48) refers to the CTC formulation for DC-OPF problem that in detail describes in [10].

\section{B. Sub-problem}

The objective function (50) checks the feasibility of network-constrained AC-OPF problem for the master problem solution by considering first and second stage network constraints. Thus, the sub-problem is formulated in (50)-(61) as below.

$\min \Phi=\sum_{n}\left(\left(\Psi_{1, n t}+\Psi_{2, n t}\right)+\left(\Psi_{1, n t}^{ \pm}+\Psi_{2, n t}^{ \pm}\right)\right)$

The first stage constraints are:

$$
\begin{gathered}
\sum_{g \in \chi_{n}} P_{g}+\sum_{m \in \Omega_{n}}\left(\left(1-\vartheta_{k}\right) f_{n m}\right)+\sum_{m \in \Omega_{n}}\left(\vartheta_{k} f_{T C S C, n m}\right) \\
+P_{f, w}=d_{n}+\Psi_{1, n t}-\Psi_{2, n t}
\end{gathered}
$$

(2)-(19)

$P_{g}=\hat{P}_{g} \rightarrow \kappa_{1, g}$

$v_{k}=\hat{v}_{k} \rightarrow \kappa_{2, k}$

$\lambda=\hat{\lambda} \rightarrow \kappa_{3}$

$f_{T C S C, n m}=\hat{f}_{T C S C, n m} \rightarrow \kappa_{4, k}$

The second stage constraints are:

$$
\begin{aligned}
\sum_{g \in \chi_{n}} P_{g}^{ \pm} & +\sum_{m \in \Omega_{n}}\left(\left(1-\vartheta_{k}\right) f_{n m}^{ \pm}\right)+\sum_{m \in \Omega_{n}}\left(\vartheta_{k} f_{T C S C, n m}^{ \pm}\right) \\
& +(1 \pm \lambda) P_{f, w}=d_{n}+\Psi_{1, n t}^{ \pm}-\Psi_{2, n t}^{ \pm}
\end{aligned}
$$

(20)-(38) 


$$
\begin{aligned}
& f_{T C S C, n m}^{ \pm}=\hat{f}_{T C S C, n m}^{ \pm} \rightarrow \kappa_{5, k}^{ \pm} \\
& P_{g}^{ \pm}=\hat{P}_{g}^{ \pm} \rightarrow \kappa_{6, g}^{ \pm} \\
\hat{\Phi} & +\sum_{g}\left(\kappa_{1, g}\left(P_{g}-\hat{P}_{g}\right)+\kappa_{6, g}^{ \pm}\left(P_{g}^{ \pm}-\hat{P}_{g}^{ \pm}\right)\right)+\sum_{k} \kappa_{2, k}\left(v_{k}-\hat{v}_{k}\right) \\
+ & \kappa_{3}(\lambda-\hat{\lambda})+\sum_{k} \kappa_{4, k}\left(f_{T C S C, n m}-\hat{f}_{T C S C, n m}\right) \\
+ & \sum_{k} \kappa_{5, k}^{ \pm}\left(f_{T C S C, n m}^{ \pm}-\hat{f}_{T C S C, n m}^{ \pm}\right) \leq 0
\end{aligned}
$$

The objective function (50) represents total active bus mismatch for first and second stage active nodal balance which should be minimized. In (50), variables $\Psi_{1, n t} / \Psi_{2, n t}$ and $\Psi_{1, n t}^{ \pm} / \Psi_{2, n t}^{ \pm}$are surplus/deficit power mismatch of the first and second stages, respectively. Constraints (51) and (57) are the same as (4) to (44), respectively. Decision variables in (53) $-(56)$ and (53) $-(56)$, are the fixed values provided by the master problem and $\kappa_{(\bullet)}^{(\bullet)}$ represents the dual variables of these constraints. If $\Phi>\varepsilon$ the feasibility benders' cut (61) will be generated and added to the master problem to mitigate the violations (i.e., (51)) in the next iteration. Noted that $\varepsilon$ is a small positive number specified by the decision maker.

\section{CASE STUdies}

To investigate the performance of the proposed AC-OPF problem with and without FRs, the modified six-bus and IEEE 118-bus systems are implemented and discussed in the following subsections $\triangleright$

\section{A. Six-Bus System}

The modified six-bus test system has been shown in Fig. 2 , which has three conventional generation units and a wind farm at bus 5 with the capacity of $100 \mathrm{MW}$. It is assumed that the forecasted WPG is equal to the nominal capacity of wind farm at Bus 5. The units and lines data are taken from [10]. The peak load is $256 \mathrm{MW}$, the share of each load at Buses 3, 4 , and 5 is 80,110 , and $66 \mathrm{MW}$, respectively. The TCSC devices have been located on Line 5-6. The minimum and maximum limits of the TCSC's reactance are $\bar{x}_{k}=-0.20 \mathrm{p} . \mathrm{u}$ and $\bar{x}_{k}=0.20$ p.u, respectively.

At first, it is assumed that the wind power output at Bus 5 is $100 \mathrm{MW}$. The cost threshold is equal to $T C_{b}=\$ 3840.415$. As previously mentioned, the cost threshold can be specified based on the conservatism levels of decision makers. Indeed, accommodation of higher uncertain WPGs will result in the higher cost thresholds. Then, the proposed AC-OPF problem with and without FRs, is solved to obtain the maximum span of the uncertainty variation range of WPGs, i.e., $\lambda$. As seen in Table 1 , in the case of no FRs, the value of $\lambda$ is 0.741 , which is taken as a reference value for comparison in the next cases. In this situation, the wind farm output is $174.1 \mathrm{MW}$ implying that it is $74.1 \mathrm{MW}$ more than the forecasted value. Indeed, for the case of ignoring FRs with $\lambda>0.741$, the proposed optimization problem turns into infeasible and there is unacceptable region for the wind uncertainty. By adding flexibility resources to the problem, as seen in Table 1 , the maximum variation range of wind uncertainty increased.
Indeed, for $\lambda>0.741$, there is a congestion in transmission lines (in particular, through Line 4-5) that limits the power flowing through lines.

By implementing the TCSC device in Line 5-6, as shown in Table 2, the active power flowing through this line has been increased from 0.084 p.u. to 0.255 p.u., while this device can effectively control the power flow over lines. Accordingly, the $\lambda$ value has been improved by $18.83 \%$ from 0.741 to 0.913 . Noted that, for $\lambda>0.913$, while TCSC reaches its control limits, its effects have been reduced.

Also, as addressed in Table 2, by opening Line 4-5 in the CTC action, the power flowing through Line 5-6 has been increased from 0.255 p.u. to 0.831 p.u. Accordingly, the $\lambda$ value has been amplified by $7.87 \%$ from 0.913 to 0.991 (as seen in Table 1).

That is, the CTC action leads to more increase in the $\lambda$ value with respect to deploying the TCSC. Hence, as can be seen in Table 1, by implementing CTC, the limitation of the TCSC is resolved and the $\lambda$ value has been more increased by $7.87 \%$.

Note that, the simultaneous utilization of TCSC and CTC, the $\lambda$ value has been increased by $31.91 \%$ while the power flowing through the Line 5-6 has been significantly increased (as shown in Table 2). That is, the robustness level of the power system operation in the presence of uncertainty can be enhanced by multiple utilization of FRs.

\section{B. The IEEE 118-Bus as a Large-Scale System}

To demonstrate the effectiveness and computational performance of the proposed framework, the simulation results of a relatively large-scale test system, i.e., IEEE 118bus system, are presented here. This test system comprises 186 lines, 91 load buses and 54 thermal units. The data of this system is taken from [15].

The peak demand is $7306 \mathrm{MW}$. Here, three wind farms with $300 \mathrm{MW}$ capacity have been installed at buses 15, 54 and 96. The four series TCSC devices are located at the heavily loaded lines (including 15-18, 54-76, 96-149, and 96150) with low capacity in the modified IEEE 118-bus. The variation range of reactance of each TCSC device is set to $90 \%$ capacitive and $40 \%$ inductive reactance of the transmission line where the TCSC devices are installed.

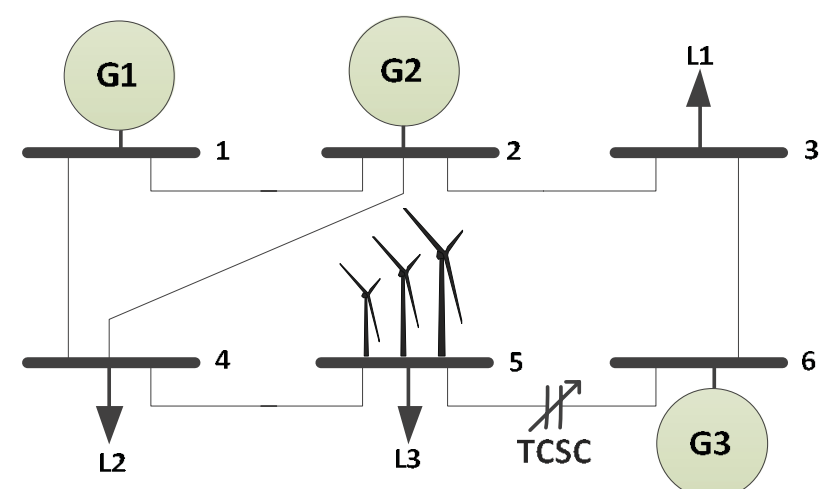

Fig. 2. The six-bus test system with a wind farm and a TCSC device. 
Table 1: The $\lambda$ value for 6-bus system.

\begin{tabular}{|c|c|c|}
\hline FRs & $\lambda$ & Computation time [s] \\
\hline No. FRs & 0.734 & 2 \\
\hline TCSC & 0.905 & 5 \\
\hline CTC & 0.987 & 7 \\
\hline $\begin{array}{c}\text { TCSC \& } \\
\text { CTC }\end{array}$ & 1.078 & 11 \\
\hline
\end{tabular}

Table 2: Active power flow (p.u.) on Lines 2-3 and 4-5

\begin{tabular}{c|c||c}
\hline \multirow{2}{*}{ FRs } & Line & Active power flow (p.u.) \\
\hline \multirow{3}{*}{ No. FRs } & $4-5$ & 0.497 \\
\cline { 2 - 3 } & $5-6$ & 0.084 \\
\hline \multirow{3}{*}{ TCSC } & $4-5$ & 0.498 \\
\cline { 2 - 3 } & $5-6$ & 0.255 \\
\hline CTC & $5-6$ & 0.831 \\
\hline $\begin{array}{c}\text { TCSC } \\
\text { \& CTC }\end{array}$ & $5-6$ & 0.921 \\
\hline
\end{tabular}

Table 3: The $\lambda$ value for IEEE 118-bus system.

\begin{tabular}{|c|c|c|}
\hline FRs & $\lambda$ & Computation time [s] \\
\hline No. FRs & 1.214 & 40 \\
\hline TCSC & 1.315 & 85 \\
\hline CTC & 1.297 & 103 \\
\hline $\begin{array}{c}\text { TCSC \& } \\
\text { CTC }\end{array}$ & 1.359 & 122 \\
\hline
\end{tabular}

The results for $\lambda$ value are given in Table 3 , the AC-OPF problem is feasible without FRs and $\lambda$ value is 1.212 . Accordingly, the value $\lambda=1.211$ has been considered as a reference value for the comparison. By comparing the performance of TCSC device with CTC for the IEEE 118bus system, as seen in Table 3 , by implementing the TCSC devices, the $\lambda$ value has been improved by $7.69 \%$ while with the CTC action the rate is about $6.34 \%$. Indeed, this result differs from the result of the previous case. Because, adding multiple TCSC will provide more flexibility in the system. Finally, again here, by concurrent operation of FRs, i.e., the TCSC devices and CTC action, the better value for $\lambda$ has been achieved, i.e., $\lambda=1.359$.

\section{CONCLUSIONS}

This paper provides a technique to solve for the maximum variation range of WPG uncertainty in an AC-OPF problem with FRs using an interval-based robust approach. Furthermore, CTC and TCSC devices are used as FRs for a more efficient utilization of the existing transmission network. In summary, the TCSC devices and CTC action can co-operatively offer significant capability in controlling the variation range of wind uncertainty. Nevertheless, it was shown that the AC-OPF formulation with FRs is a MINLP problem for which a solution is an intractable task. Therefore, BDA was used to decompose and simplify the problem. The simulation results showed that the BDA was able to find the optimal solution for a six-bus and the IEEE 118-bus test systems.

\section{ACKNOWLEDGMENT}

J.P.S. Catalão acknowledges the support by FEDER funds through COMPETE 2020 and by Portuguese funds through FCT, under SAICT-PAC/0004/2015 (POCI-01-0145FEDER-016434) and 02/SAICT/2017 (POCI-01-0145FEDER-029803).

\section{REFERENCES}

[1] A. Nikoobakht, and J. Aghaei, "IGDT-based robust optimal utilisation of wind power generation using coordinated flexibility resources," IET Renewable Power Generation, vol. 11, no. 2, pp. 264-277, 2016.

[2] S. Gasperic, and R. Mihalic, "The impact of serial controllable FACTS devices on voltage stability," International Journal of Electrical Power \& Energy Systems, vol. 64, pp. 1040-1048, 2015.

[3] A. Nikoobakht, J. Aghaei, T. Niknam, M. Shafie-khah, and J. P. Catalão, "Smart Wire Placement to Facilitate Large-Scale Wind Energy Integration: An Adaptive Robust Approach," IEEE Transactions on Sustainable Energy, 2018.

[4] R. Yang, and G. Hug-Glanzmann, "Optimal usage of transmission capacity with FACTS devices in the presence of wind generation: a two-stage approach." pp. 1-7.

[5] A. Nikoobakht, J. Aghaei, and M. Mardaneh, "Securing highly penetrated wind energy systems using linearized transmission switching mechanism," Applied Energy, vol. 190, pp. 1207-1220, 2017.

[6] A. Nikoobakht, J. Aghaei, R. Khatami, E. Mahboubi-Moghaddam, and M. Parvania, "Stochastic flexible transmission operation for coordinated integration of plug-in electric vehicles and renewable energy sources," Applied Energy, vol. 238, pp. 225-238, 2019.

[7] W. Lin, J. Wen, S. Cheng, and W.-J. Lee, "An investigation on the active-power variations of wind farms," Industry Applications, IEEE Transactions on, vol. 48, no. 3, pp. 1087-1094, 2012.

[8] P. Chen, P. Siano, Z. Chen, and B. Bak-Jensen, "Optimal allocation of power-electronic interfaced wind turbines using a genetic algorithmmonte carlo hybrid optimization method," Wind Power Systems, pp. 123: Springer, 2010.

[9] M. Sahraei-Ardakani, and K. W. Hedman, "Computationally Efficient Adjustment of FACTS Set Points in DC Optimal Power Flow with Shift Factor Structure," IEEE Transactions on Power Systems, vol. 32, no. 3, pp. 1733-1740, 2017.

[10] A. Khodaei, and M. Shahidehpour, "Transmission switching in security-constrained unit commitment," Power Systems, IEEE Transactions on, vol. 25, no. 4, pp. 1937-1945, 2010.

[11] A. Nikoobakht, M. Mardaneh, J. Aghaei, V. Guerrero-Mestre, and J. Contreras, "Flexible power system operation accommodating uncertain wind power generation using transmission topology control: an improved linearised AC SCUC model," IET Generation, Transmission \& Distribution, vol. 11, no. 1, pp. 142-153, 2017.

[12] A. Nikoobakht, J. Aghaei, T. Niknam, V. Vahidinasab, H. Farahmand, and M. Korpås, "Towards robust OPF solution strategy for the future AC/DC grids: case of VSC-HVDC-connected offshore wind farms," IET Renewable Power Generation, vol. 12, no. 6, pp. 691-701, 2018.

[13] J. Aghaei, A. Nikoobakht, P. Siano, M. Nayeripour, A. Heidari, and M. Mardaneh, "Exploring the reliability effects on the short term AC security-constrained unit commitment: A stochastic evaluation," Energy, vol. 114, pp. 1016-1032, 2016.

[14] F. Qiu, and J. Wang, "Chance-constrained transmission switching with guaranteed wind power utilization," Power Systems, IEEE Transactions on, vol. 30, no. 3, pp. 1270-1278, 2015.

[15] motor.ece.iit.edu/data/SCUC_118. 